\title{
Intersensory concepts in children
}

\author{
FELIX E. GOODSON, MICHAEL P. SILVER, JOSEPH SCHUMAKER, \\ and BETTE M. BUNTING \\ DePauw University, Greencastle, Indiana 46135
}

\begin{abstract}
This experiment was designed to determine whether intersensory transfer can be demonstrated using form (stars, squares, circles, and parallelograms) concepts as mediators. It was hypothesized that the association of certain sounds with visually presented forms would significantly influence the association of these sounds with the same or different forms when tactually presented. Three groups of subjects were used. During training trials, both experimental groups learned the same sound/form associations (with the forms being visually presented). During test trials, both experimental groups were presented the forms tactually, with Experimental Group 1 being tested with the same sound/form pairings given during training trials and Experimental Group 2 being tested with different sound/form pairings. The control group was exposed to a completely different set of stimuli (four new sounds and four line drawings) during training trials and exactly the same conditions as Experimental Group 1 during the test trials. The results indicated that form concept mediation across modalities can result in considerable transfer.
\end{abstract}

Intersensory transfer provides a functional simplification of an individual's environment. Thus, for instance, one may recognize an apple from its taste, touch, feel, or visual appearance. The inputs from these different modalities have become integrated and represented by a single concept, "apple."

The present study was designed to determine whether intersensory transfer can be demonstrated using form concepts as mediators. Hypothesis: The association of certain sounds with visually presented forms will significantly influence the association of these sounds with the same or different forms when tactually presented.

\section{METHOD}

\section{Subjects}

Forty-five 8-year-old children matched into three groups on the basis of age, sex, and intelligence were used.

\section{Apparatus}

The display apparatus included a $3 \times 3 \mathrm{ft}$ plywood board with an opening, $5 \mathrm{in}$. high and $10 \mathrm{in.}$ long, located $1 \mathrm{in}$. from the bottom in the center of the board. A hinged board, also $5 \mathrm{in}$. high and $10 \mathrm{in}$. long, could be lowered to expose the visual stimuli and raised to expose two rubber irises, $1 \mathrm{in}$. apart and 3 in. in diameter, through which the subject could thrust his hands to feel the forms when they were tactually presented.

Two $3 \times 4$ in. reinforcement windows were located side by side $6 \mathrm{in}$. above the hinged board. The left window had a green light that projected through a stencil reading "right," and the right window had a red light that projected through a stencil reading "wrong."

The sound stimuli were recorded and then spliced into a master tape in order of presentation. Three master tapes were used: one for the training trials for Experimental Groups 1 and 2 that consisted of $5 \mathrm{sec}$ of white noise, ringing bells, tones and

Reprint requests should be sent to the first author, Department of Psychology, DePauw University, Greencastle, Indiana 46135. clicks; one for training trials for the control group that consisted of $5 \mathrm{sec}$ of sawing, drumbeats, water flowing, and dog barking; and one for the test trials for all groups that held the same stimuli given to the experimental groups during training, in a different order.

The forms (stars, circles, squares, and parallelograms) were made of .5-in. plywood and were approximately 1 in. in diameter. They were painted yellow and mounted on a small gray piece of plywood, $5 \times 5.5 \mathrm{in}$. The visual display cards for the control group training trials consisted of four horizontal 4-in. black drawings (a series of loops, angles, triangles, and two parallel lines .125 in. apart).

\section{Procedure}

Matching treatment. The subjects were matched into two experimental groups and one control group on the basis of sex, age in months, and intelligence (based on Otis Quick Scoring, Grades 1.5-4.0). The subjects in the two experimental groups were also matched on the basis of their performance during training trials. A matching-in-process technique was used to pair subjects in the two experimental groups on the basis of their performance during training trials. This was done to insure that the two groups would have approximately the same ability for learning associations.

Training trials. The subjects were brought individually into the experimental room and given the following instructions: "Each of these forms has a sound that goes with it. When you see two of these forms here, you will hear a sound which will continue for $5 \mathrm{sec}$. We want you to point to which form you think goes with that sound. If you are right, you will see a green light here. If you are wrong, you will see a red light here. We want you to see how fast you can learn which form goes with which sound."

Two visual forms were placed in the display window, and the corresponding sound was presented for $5 \mathrm{sec}$. The subject pointed to the form he thought went with the sound, and his response was either corrected or approved by means of the green and red lights. The presentations were randomly established but used in the same order for every subject in both experimental groups. Each form was paired with each other form in both right and left positions an equal number of times. Training was continued until the subject reached the criterion of two correct sequences in a row. If the subject failed to reach criterion in 72 trials, he was eliminated. Four subjects 
in the experimental groups failed to reach criterion, leaving 13 subjects in each group.

The control group was given exactly the same treatment as the experimental groups, except that four different visual stimuli and four different sounds were used.

Test trials. After a 5-min rest, each subject in every group was given the following instructions: "Each time you hear a sound, stick your hands through these holes and feel the forms on the other side. Feel each object and try to tell which one goes with the sound. When you have made your choice, let go of the other one and keep the one you think is correct in your hand. As soon as I have taken this form from you, remove your hands from the holes. If you have made the correct choice, you will see the green light; if you are wrong, you will see the red light."

Exactly the same procedure was followed during test trials that had been followed during training trials, except that: (1) all subjects were run until they reached criterion; (2) all forms were presented tactually; (3) only the order of presentation was changed for Experimental Group 1; (4) both the order of presentation and the sound/form associations were changed for Experimental Group 2; and (5) the control group was given the same treatment as Experimental Group 1.

\section{RESULTS}

The difference between the two experimental groups on the test trials yielded a $t$ of 4.67 , with 2.68 needed at the .01 level. This finding clearly supports the hypothesis that form concept mediation can result in transfer across modalities. The difference between the control group and Experimental Group 2 was also significant, with a $\mathrm{t}$ of 3.83 . The $\mathrm{t}$ for the control group vs. Experimental Group 1 approached but did not reach significance.

The pronounced negative transfer effects found in Experimental Group 2 may have been due to two factors. First, it was necessary for the subjects in this group to unlearn the first and competing association, and second, it was necessary for them to learn the new association.

(Received for publication January 31, 1982.) 